\title{
Molecular docking and binding interaction between psychedelic drugs and human serum albumin
}

\author{
HOSSEIN KHASTAR ${ }^{2}$, Kobra Foroughi ${ }^{1}$, SEYed SHaHrokH Aghayan ${ }^{2}$, \\ MARYAM YARMOHAMMADI ${ }^{2}$, MOSLEM JAFARISANI ${ }^{2 *}$ \\ ${ }^{1}$ Student Research Committee, School of Medicine, Shahroud, University of Medical Sciences, Shahroud, Iran \\ ${ }^{2}$ School of Medicine, Shahroud University of Medical Sciences, Shahroud, Iran
}

\begin{abstract}
Drug-plasma protein interaction is a critical concern in monitoring drug circulation and drug-drug interactions. The present study aimed to investigate the interaction of psychedelic drugs such as lysergic acid diethylamide (LSD), dimethyltryptamine (DMT), 2,5-dimethoxy-4-iodoamphetamine (DOI), psilocybin, psilocin, and mescaline with human serum albumin (HSA). The 3D structures of LSD, DMT, DOI, psilocybin, psilocin, mescaline, and albumin were obtained from the structural databases (www.rcsb.org, https://pubchem.ncbi.nlm.nih.gov/ compound). The structures were then prepared for molecular docking analysis by Autodock Vina software. Ultimately, the binding energies between docked HSA and psychedelic drugs were calculated, and their interactions were predicted. It was found that the psychedelic drugs can interact with HSA in the active site and the best minimum binding energies of $-7.6 \mathrm{kcal} / \mathrm{mol}$ and $-6.5 \mathrm{kcal} / \mathrm{mol}$ were shown by LSD and psilocybin, respectively. Our results indicated that all psychedelic drugs tested could interact with HSA at subdomains IA and IB. The structural properties of the drugs affect their interaction sites and binding energies. It was concluded that albumin, as the most abundant protein of the serum, could act as the biodistributor of psychedelic drugs.
\end{abstract}

Key words: human serum albumin, biodistribution, psychedelic drugs, molecular docking

\section{Introduction}

The nature of drug-protein interaction gives new opportunities for the development of novel drugs, which has vital implications on the drug tissue disposition and metabolic rate. Various drugs such as ibuprofen, diazepam, and warfarin can bind reversibly to plasma proteins (Fender and Dobrev, 2019). The effect of a drug depends on the availability of the free drug in plasma in order to bind to the receptor sites and elicit pharmacological response. Studies have shown that drugs are distributed in blood circulation, either in free form or in the form of a complex with plasma proteins; therefore, the binding of drugs to plasma proteins is a very important factor that determines the effect of pharmacokinetics and pharmacodynamics of drugs (He and Carter, 1992; Hegde et al., 2011; Jafarisani et al., 2018; Khalili et al., 2017; Ran et al., 2007).
Human serum albumin (HSA) is the most abundant protein in serum and plasma. It plays an essential role as a carrier of blood molecules such as free fatty acids and hormones and some drugs. Moreover, HSA is used as a source of distribution of amino acids and plays a critical role in the generation and preservation of osmotic pressure in blood flow (Lee and $\mathrm{Wu}, 2015$ ). In addition, HSA binding can increase the half-life of the circulating drugs. HSA is a globular protein composed of three homologous domains (I-III) (Meloun et al., 1975; Phillips et al., 1989; Sudlow et al., 1975), and it is the best-studied model to explain the ligand delivery process in vivo because the interaction of any toxic substance with HSA affects the transportation of nutrients and drugs (Khalili et al., 2017). Hence, the understanding of interaction mechanisms between toxic chemicals and HSA is very crucial for toxicology research (Hou et al., 2015). Recent

\footnotetext{
* Corresponding authors: School of Medicine, Sharhoud University of Medical Sciences, Shahroud, Iran; e-mail: moslem.jafarisani@shmu.ac.ir
} 
studies have reported the binding of organic contaminants or toxins to HSA, for example, atrazine and methyl parathion, by using experimental and computational approaches (Bertel et al., 2013; Purcell et al., 2001; Shooshtary et al., 2015; Silva et al., 2004).

Serotonergic hallucinogens (psychedelics) are potent psychoactive materials that modify thoughts and feelings of an individual and influence various cognitive behaviors (Nichols, 2016). They are considered to be physiologically safe for routine administration and do not lead to dependence or addiction (Nichols, 2016). Chan and Mendelson (2014) categorized serotonergic hallucinogens in three groups according to their chemical structure: tryptamines such as N,N-dimethyltryptamine (DMT), psilocybin, and psilocin; lysergamines such as lysergic acid diethylamide (LSD); and phenethylamines such as 2,5-dimethoxy-4-iodoamphetamine (DOI) and mescaline (Chan and Mendelson, 2014). Researchers have attempted to induce the hallucinogen effects through the serotonergic system, and there is a small but increasing amount of evidence showing that they may have therapeutic use in treating anxiety disorders and depression (Baumeister et al., 2014; Jang et al., 2010; Liechti, 2017; Ly et al., 2018). Hallucinogens are nonpolar compounds and thus their transportation in blood is difficult; hence, they need to be bound to carriers in order to enable them to reach the brain. As a general transporter present in the blood, HSA has been suggested to be used for this purpose (Sherwood et al., 2020; Zielinski et al., 2020).

The present study aimed to investigate the interaction of psychedelic drugs such as LSD, DMT, DOI, psilocybin, psilocin, and mescaline with HSA. By using similar in silico approaches, our present study attempted to assess whether HSA can be used as a biodistributor for psychedelics. To this end, various state-of-the-art bioinformatics tools were used to analyze the possible interactions between HSA and psychedelic drugs and the possible consequences on their pharmacokinetic and pharmacodynamic properties, including adsorption, distribution, metabolism, and excretion.

\section{Methods}

\section{$3 D$ structures}

The crystallographic structure of HSA was obtained from the Protein Data Bank (PDB), with the PDB ID of $1 \mathrm{~N} 5 \mathrm{U}$. This structure has a resolution of $1.9^{\circ} \mathrm{A}$. The

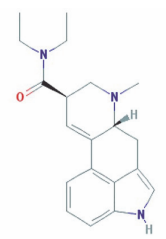

LSD (1)

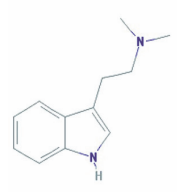

DMT (4)

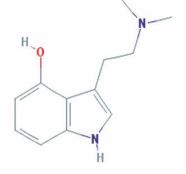

Psilocin (2)

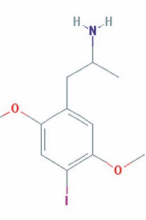

DOI (5)

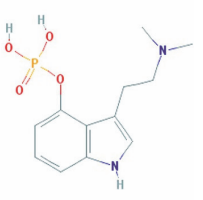

Psilocybin (3)

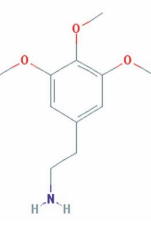

Mescaline (6)
Fig. 1. 1) Structures of LSD, 2) psilocin, 3) psilocybin, 4) DMT, 5) DOI, and 6) mescaline obtained from the PubChem database

PDB file for HSA was edited to exclude the unnecessary ligands and water molecules. The ViewerLite software (Version 5.0) was used to delete unwanted molecules.

The 3D structures of psychedelic drugs (LSD, DMT, DOI, psilocybin, psilocin, and mescaline, shown in Figure 1 and Table 1) were extracted from the PubChem database at https://pubchem.ncbi.nlm.nih.gov/compound (Kim et al., 2016). The 3D structures were saved in sdf file format and were converted to the pdb format by the ViewerLite software.

\section{Molecular docking}

The Autodock Vina molecular docking software was used to analyze the possible orientations for the interactions between HSA and psychedelic drugs. The Autodock Vina software is a program for molecular docking and virtual screening analysis; it has a new scoring function that improves the speed and accuracy of docking as compared to Autodock (Trott and Olson, 2010). The Autodock Vina system requires that the receptor and ligand files are formatted in the PDBQT molecular structure file format. The MGLTools (Version 1.5.6) was used for the preparation of file format conversion and the HSA 3D structure for docking analysis by adding hydrogen atoms and merging all nonpolar hydrogens. The rotatable bounds were defined and allowed to have rotation. The grid box was defined according to the entire spacing structure. Autodock Vina was also used for docking ligands in HSA. In addition, 10 docked conformations with minimum binding energies were listed for each run with the Iterated Local Search global opti- 
Table 1. Binding energies between HSA and LSD, DMT, psilocybin, psilocin, DOI, and mescaline. Lower energies indicate more stable and strong interaction between HSA and ligands. All drugs (defined with CID) were obtained from the PubChem database

\begin{tabular}{l|c|c|l|l}
\hline Structure & $\begin{array}{c}\text { PubChem } \\
\text { CID }\end{array}$ & $\begin{array}{c}\text { Binding } \\
\text { affinity } \\
{[\mathrm{kcal} / \mathrm{mol}]}\end{array}$ & Hydrophobic interaction & \multicolumn{1}{c}{$\begin{array}{c}\text { H-bond interaction } \\
\text { (hydrogen bond length) } \\
{\left[{ }^{\circ} \mathrm{A}\right]}\end{array}$} \\
\hline LSD & 5661 & -7.6 & Asp108, Ser193, Cys249 & Tyr148 (3.04), Gln196 (3.03) \\
\hline DMT & 6089 & -5.1 & Asp108, Tyr148, Ser193, Gln196, Arg197 & - \\
\hline Psilocybin & 10624 & -6.5 & Leu115, Arg117, Pro118, Tyr138, Ile142, Tyr161, Arg186 & - \\
\hline Psilocin & 4980 & -6.0 & Val46, Glu45, Glu48, Phe49, Leu69 & Lys73 (3.30) \\
\hline DOI & 1229 & -6.2 & Arg117, Leu135, Tyr138, Ile142, Tyr161, Arg117 & - \\
\hline Mescaline & 4076 & -5.4 & Glu45, Val46, Phe49, Leu69, Phe70, Asp72 & Lys73 (2.91) \\
\hline
\end{tabular}

A

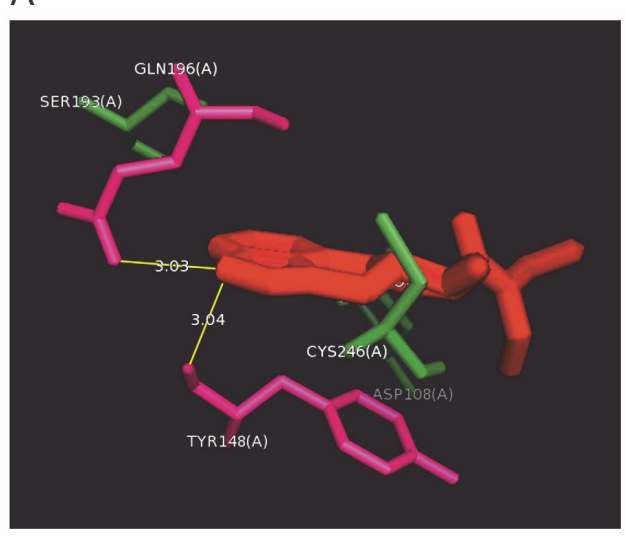

B

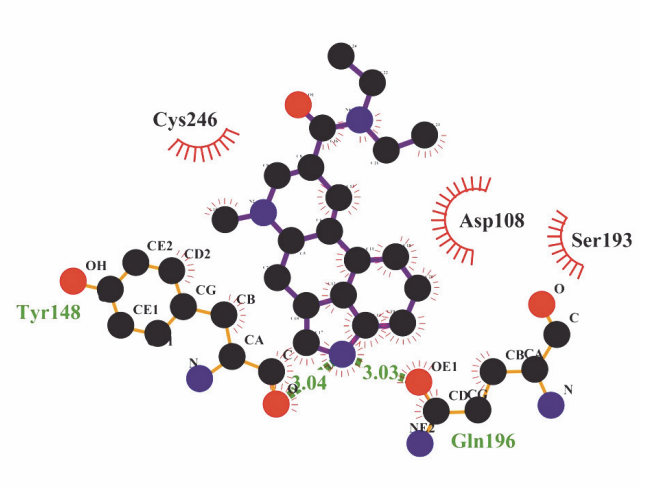

Fig. 2. A) Structure of the LSD-HSA complex as a mesh surface image generated by PyMOL, B) 2D scheme of the interactions between LSD and HSA generated by LIGPLOT

mizer algorithm. Eventually, the binding energies for the interaction between the HSA and psychedelic drugs were calculated. The PyMOL software was used to obtain the pdb complex file.

\section{$2 D$ and $3 D$ interaction plots}

The schematic diagrams of detailed protein-ligand interactions were generated using the LigPlus program (Version 1.3.6). This program indicated the potential interactions between the ligand molecules and HSA residues. In addition, the PyMOL software was used for $3 \mathrm{D}$ interaction plot.

\section{Results}

In the present study, the interaction and binding energies of psychedelic drugs such as LSD, DMT, DOI, psilocybin, psilocin, and mescaline with HSA were determined using in silico molecular docking. An overview of the docking interactions of the protein and ligands is presented in Table 1.

\section{Interaction of LSD with the binding site of $\mathrm{HSA}$}

The program predicted $-7.6 \mathrm{kcal} / \mathrm{mol}$ of the minimum binding energy for the complex of LSD with HSA. LSD interacted with the hydrophobic residues of HSA, including Asp108, Ser193, and Cys249. Furthermore, LSD was found to interact with Tyr148 and Gln196 of HSA through hydrogen bonds. Detailed interactions of LSD with the binding site of HSA is shown in Figure 2.

\section{Docking results of DMT, psilocybin, and psilocin}

The results of the docking of DMT to HSA showed that the minimum binding energy was $-5.1 \mathrm{kcal} / \mathrm{mol}$. It was observed that hydrophilic residues of has, including Asp108, Tyr148, Ser193, Gln196, and Arg197, interact with DMT through Van der Waals binding. The 
A

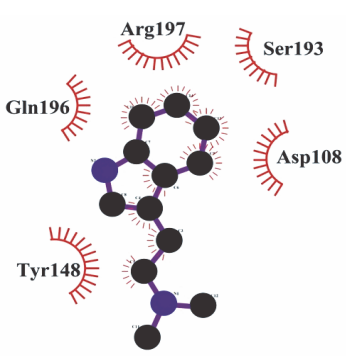

B

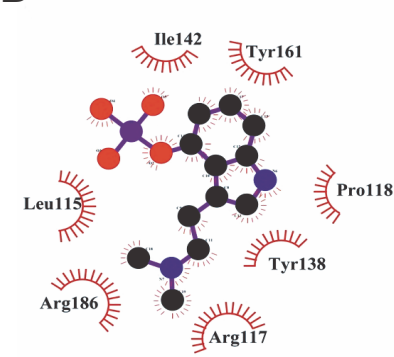

DMT

C

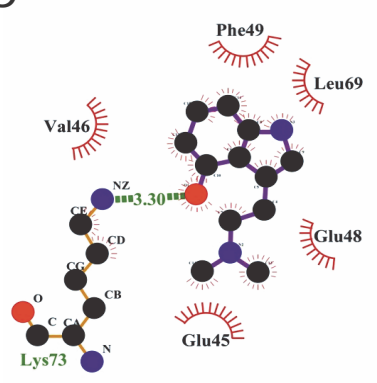

Psilocybin
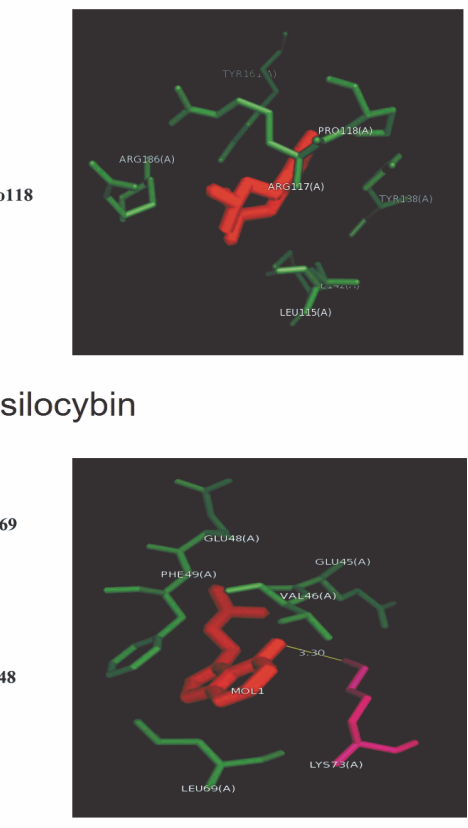

Psilocin

Fig. 3. A) 2D and 3D schemes of the interactions between DMT, B) psilocybin, and C) psilocin with HSA generated by LIGPLOT (2D) and PyMOL (3D)

detailed interaction between DMT and HSA is shown in Figure 3A.

The docking results of psilocybin to HSA showed that the minimum binding energy was $-6.5 \mathrm{kcal} / \mathrm{mol}$. The interaction was mediated by hydrophobic residues including Leu115, Pro118, and Ile142, and hydrophilic residues including Arg117, Tyr138, Tyr161, and Arg186 through Van der Waals binding. Detailed interactions of this compound with HSA are shown in Figure 3B.

As shown in Figure 3C, the minimum binding energy of psilocin and HSA was $-6.0 \mathrm{kcal} / \mathrm{mol}$, and the hydrophobic and hydrophilic residues of HSA, including Val46, Glu45, Glu48, Phe49, and Leu69, were involved in this interaction by Van der Waals binding. In addition, psilocin interacted with Lys73 through a hydrogen bond.

\section{Docking results of DOI and mescaline}

The docking experiments indicated that the minimum binding energy of a complex formed by DOI and HSA was $-6.2 \mathrm{kcal} / \mathrm{mol}$. This interaction included the hydrophobic residues Leu135 and Ile142 and the hydrophilic residues Arg117, Tyr138, Tyr161, and Arg117 of HSA. More information on the interaction between DOI and HSA is given in Figure 4A.

Our docking results also showed that mescaline can harness HSA by binding to one of its subdomains which is located in binding site 1 with the minimum binding energy of $-5.4 \mathrm{kcal} / \mathrm{mol}$. As shown in Figure 4B, the hydrophobic residues of HSA that interacted with mescaline were Val46, Phe49, Leu69, and Phe70 and the hydrophilic residues were Glu45 and Asp72 as well as Lys73 through hydrogen bonding.

\section{Interaction of active site of $\mathrm{HSA}$ with psychedelic drugs}

The superimposition of the docking poses of HSA and the psychedelic drugs in the binding site of HSA is shown in Figure 5. As illustrated in the figure, we observed that the drugs could bind to HSA in the IB site located in Domain I.

\section{Discussion}

Psychedelic drugs including mescaline, psilocybin, DMT, and LSD are used by humans because of their capacity to elicit profound alterations in attention, passion, and cognitive processes. Swanson (2018) reported that psychedelic drugs repress the core brain mechanism that regularly perform to conquer or filter or constraint psychic aspects into an evolutionarily adaptive container. In addition, the author showed that this core brain mechanism can function pathologically, thereby restricting the perspicacity, sensation, and perception of an individual. Moreover, psychedelic phenomena and symptoms of chronic psychoses participate in representative elements because they both involve circumstances of relatively unconstrained mental processes (Swanson, 2018). Contrary to the increasing information on the neural response mechanisms associated with the acute effects of these drugs, the consequences of the maintained use of psychedelic drugs on the human brain remain mostly unexplored (Bouso et al., 2015). 

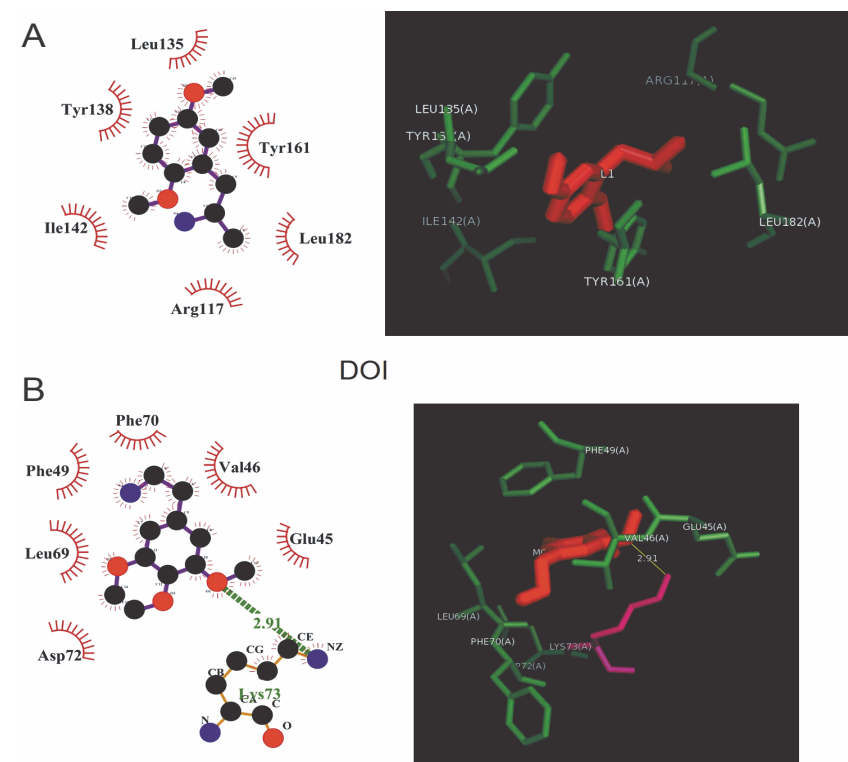

DOI

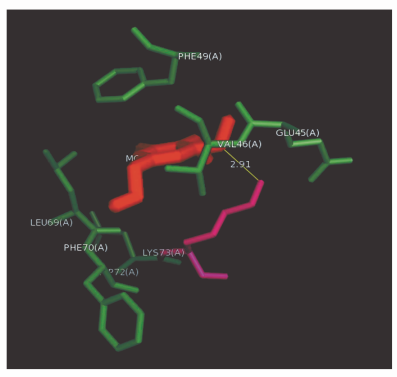

Mescaline

Fig. 4. A) $2 \mathrm{D}$ and 3D schemes of the interactions between DOI and B) mescaline with HSA generated by LIGPLOT (2D) and PyMOL (3D)

Molecular docking has become a commonly used powerful tool for drug discovery. The molecular docking strategy can be used to model the interaction between a small molecule and a protein at the atomic level, which allows to describe the functions of small molecules in the binding sites of target proteins and to illustrate basic biochemical processes. In molecular docking, the purpose is to predict the binding and interactions between two different molecules (Banaganapalli et al., 2019). Currently, many different docking programs are available, such as Autodock, Autodock Vina, and Molergo (Uciechowska-Kaczmarzyk et al., 2019), which use several approaches to execute these interactions. Molecular docking enables to assess binding potentiality between molecules and proteins without difficult and/or expensive laboratory work (McConkey et al. 2002; Meng et al., 2011). On the other hand, performing an absorption, distribution, metabolism, toxicity, and excretion (ADMTE) study could add valuable knowledge from the perspective of drug development. Examining the ADMTE features of drug candidates is therefore considered imperative. The binding of therapeutic agents with serum components could significantly add to their biodistribution as an ADMTE property. Impaired biodistribution of drug candidates is one of the challenges for developing therapeutic factors (Khalili et al., 2017).

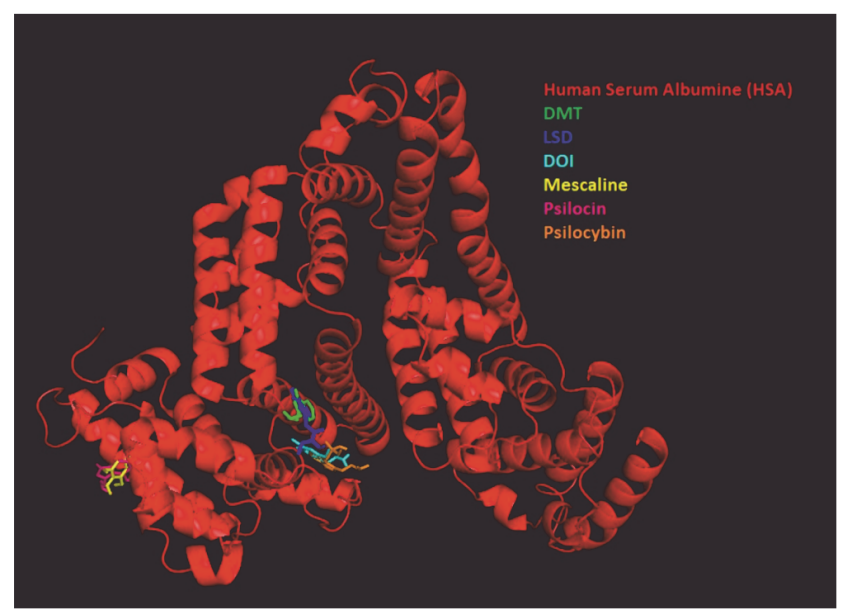

Fig. 5. The superimposition of the docking poses of psychedelic drugs in the binding site of HSA generated by PyMOL

HSA is a common essential protein in serum, which has different functions including the binding and transport of lipophilic xenobiotics and numerous endogenous substrates (Matsuda et al., 2014). The protein is composed of three homologous domains (I, II, and III), which are further subdivided into pairs of subdomains (DIA, DIB, DIIA, DIIB, DIIIA, and DIIIB) (Abou-Zied and Al-Shihi, 2008). Crystallographic studies conducted on ligand-HSA interaction have reported the molecular features of the binding (Brunmark et al., 1997; Dockal et al., 1999). In domain I, fatty acid binding site 1 , free cysteine (C34), and drug binding site 3 are located. The fatty acid site lies between DI and DII; the metal binding site is located between DIA and DIIA subdomains; and DII comprises the drug binding site 1 (Sudlow's site 1) and fatty acid sites 6 and 7. DIII holds fatty acid binding sites 3 and 4 , while the drug binding site 2 (Sudlow's site 2) is located in DIIIA. The fatty acid binding site 5 that binds to heterocyclic compounds with a negative charge is located in DIIIB. (Karimi et al., 2016). The construction of site IIIA (indole-benzodiazepine site) is topologically similar to IIIB, but the compounds that bind at this site (e.g., NSAIDs) typically contain a peripheral negative charge (Chan and Mendelson, 2014). A third binding pocket within subdomain ID (site IB) was identified as the primary binding site of a bilirubin photoisomer, hemin, a sulfonamide derivative, and the steroid antibiotic fusidic acid (Ghuman et al., 2005). Crystallographic studies have also shown that the large crevice of subdomain IB harbors secondary binding sites for some additional compounds (Brunmark et al., 1997; Doc- 
kal et al., 1999; Zsila, 2013). Zsila (2013) showed that subdomain IB can be considered as the third major drugbinding region of HSA that has promiscuous ligand recognition ability. Additionally, subdomain IB is allosterically coupled with Sudlow's site, the ligand binding of which was shown to alter the HSA binding mode and affinity of biliverdin and hemin (Zsila, 2013).

Chaves et al. (2015) studied the interaction between HSA and pheophytin. The docking results showed that pheophytin interacts through hydrogen bonds with three lysine and one arginine residues. The ligand also interacts with HSA through Leu197, Phe205, Ala209, Leu346, and Val481 residues, including the fluorophore Trp214. They concluded that pheophytin can interact with HSA with a binding energy of $-6.2 \mathrm{kcal} / \mathrm{mol}$ (Chaves et al., 2015) in the binding site located in Sudlow's site 1. In this regard, this interaction could quench the florescence of HSA in the binding state, which is not similar to ducked drugs tested here. Because of their structures, the tested drugs could not interact with Trp214; thus, it is unlikely they can act as a quencher.

In another study, Arash Hasanzadeh et al. (2017) investigated the interaction between iohexol and HSA and suggested that the binding site of iohexol to HSA was probably located on sites I and II and could bind to HSA with a binding energy of $-5.8 \mathrm{kcal} / \mathrm{mol}$ (Hasanzadeh et al., 2017). Shalbafan and Behbehani proposed that methotrexate interacts with HSA through hydrogen bonds with one lysine, two arginines, one asparagine, and one glutamine residues, and the main intermolecular interactions occurred in the subdomain IIA interaction cavity called Sudlow's site 1 (Shalbafan and Rezaei Behbehani, 2018). The binding of ponatinib, as an influential anticancer agent, to HSA occurred through Sudlow's site I (Tayyab et al., 2019). In addition, Fliszár-Nyúl et al. (2019) have demonstrated a strong interaction between alternariol and HSA, which involves Sudlow's site I as a high-affinity binding site in HSA.

In the present study, the interaction of psychedelic drugs with HSA was investigated. The results revealed that all these drugs can bind to the two active sites of HSA with different affinities and binding energies depending on their hydrophobic properties according to a previous study (Sherwood et al., 2020). Because of the hydrophobic characteristics of these drugs, it seems that HSA could act as their powerful transporter in blood. Our study showed that hydrophobic groups present on a drug molecule ensure more effective binding energies of the tested drugs to HSA. As shown in the Results section, the four compounds LSD, DMT, DOI, and psilocybin with affinity binding energies of $-7.6,-5.1,-6.2$, and $-6.5 \mathrm{kcal} / \mathrm{mol}$, respectively, may interact with HSA through DIB subdomain that contains drug site 3 . These binding energies are sufficient for binding to HSA in blood and for drug distribution as they ensure stable but reversible binding. In addition, the two other compounds, namely psilocin and mescaline, with binding energies of -6.5 and $-5.4 \mathrm{kcal} / \mathrm{mol}$, respectively, interact with HSA through DIA subdomain, which can affect drug binding site 1 and Sudlow's site 1 in the DIIA subdomain of HSA. Moreover, all the studied drugs were predicted to show no interaction with Trp214 of HSA, which is located in Sudlow's site 1; thus, they cannot act as quenchers.

\section{Conclusions}

In summary, the results of the present study indicated that psychedelic drugs can interact with HSA molecule in its active site which is located in domain 1 of HSA. The best minimum binding energies of $-7.6 \mathrm{kcal} / \mathrm{mol}$ and $-6.5 \mathrm{kcal} / \mathrm{mol}$ were determined for LSD and psilocybin, respectively. The results indicated that all the tested psychedelic drugs could interact with HSA in subdomains IA and IB. The structural properties of the drugs, such as hydrophobicity and hydrophilicity, can affect HSA folding and structure. It was concluded that albumin, as the most abundant protein of serum, could serve as the biodistributor of psychedelic drugs. More studies are needed to explore the binding mechanisms of the tested ligands to HSA. Spectroscopic methods are more powerful to identify mechanisms of interaction as binding might be static or dynamic, which depends on the HSA residues involved in these interactions.

\section{Acknowledgments}

This study was supported and funded by Grant No. 9751 from Shahroud University of Medical Sciences.

\section{References}

Abou-Zied O.K., Al-Shihi O.I. (2008) Characterization of subdomain iia binding site of human serum albumin in its native, unfolded, and refolded states using small molecular probes. J. Amer. Chem. Soc. 130(32): 10793-10801.

Banaganapalli B., Morad F.A., Khan M., Kumar C.S., Elango R., Awan Z., Shaik N.A. (2019) Molecular docking. Essentials of bioinformatics. Vol. I. Springer: 335-353. 
Baumeister D., Barnes G., Giaroli G., Tracy D. (2014) Classical hallucinogens as antidepressants? A review of pharmacodynamics and putative clinical roles. Ther. Adv. Psychopharm. 4(4): 156-169.

Bertel T.F., Ling R., Stald G. (2013) Mobile communication in the age of smartphones. Process of Domestication and Redomestication Hgv University of Copenhagen Online verfügbar unter en itu $\mathrm{dk} /{ }^{\sim} /$ media/EN/Research/PhDProgramme/PhD-defences/2013/PublicversionBertel2013 MobileCommunicationintheAgeofSmartphonespdf pdf

Bouso J.C., Palhano-Fontes F., Rodríguez-Fornells A., Ribeiro S., Sanches R., Crippa J.A.S., Hallak J.E.C., de Araujo D.B., Riba J. (2015) Long-term use of psychedelic drugs is associated with differences in brain structure and personality in humans. Eur. Neuropsychopharmacol. 25(4): 483-492.

Brunmark P., Harriman S., Skipper P.L., Wishnok J.S., Amin S., Tannenbaum S.R. (1997) Identification of subdomain ib in human serum albumin as a major binding site for polycyclic aromatic hydrocarbon epoxides. Chem. Res. Toxicol. 10(8): 880-886.

Chan R.H.A., Mendelson J.E. (2014) Chapter seventeen - hallucinogens. [in:] The effects of drug abuse on the human nervous system. Ed. Madras B., Kuhar M. Boston. Academic Press: 533-552.

Chaves O.A., Amorim A.P.dO., Castro L.H., Sant'Anna C.M.R., De Oliveira M.C., Cesarin-Sobrinho D., Netto-Ferreira J.C., Ferreira A.B. (2015) Fluorescence and docking studies of the interaction between human serum albumin and pheophytin. Molecules 20(10): 19526-19539.

Dockal M., Carter D.C., Rüker F. (1999) The three recombinant domains of human serum albumin structural characterization and ligand binding properties. J. Biol. Chem. 274(41): 29303-29310.

Fender A.C., Dobrev D. (2019) Bound to bleed: how altered albumin binding may dictate warfarin treatment outcome. Elsevier.

Ghuman J., Zunszain P.A., Petitpas I., Bhattacharya A.A., Otagiri M., Curry S. (2005) Structural basis of the drug-binding specificity of human serum albumin. J. Mol. Biol. 353(1): 38-52.

Hasanzadeh A., Dehghan G., Shaghaghi M., Panahi Y., Jouyban A., Yekta R. (2017) Multispectral and molecular docking studies on the interaction of human serum albumin with iohexol. J. Mol. Liq. 248: 459-467.

He X.M., Carter D.C. (1992) Atomic structure and chemistry of human serum albumin. Nature 358: 209.

Hegde A.H., Sandhya B., Seetharamappa J. (2011) Evaluation of binding and thermodynamic characteristics of interactions between a citrus flavonoid hesperitin with protein and effects of metal ions on binding. Mol. Biol. Rep. 38(8): 4921-4929.

Hou H., Qu X., Li Y., Kong Y., Jia B., Yao X., Jiang B. (2015) Binding of citreoviridin to human serum albumin: multispectroscopic and molecular docking. BioMed Res. Int. 2015: 162391-162391.

Jafarisani M., Bathaie S.Z., Mousavi M.F. (2018) Saffron carotenoids (crocin and crocetin) binding to human serum al- bumin as investigated by different spectroscopic methods and molecular docking. J. Biomol. Struct. Dynam. 36(7): 1681-1690.

Jang S.W., Liu X., Yepes M., Shepherd K.R., Miller G.W., Liu Y., Wilson W.D., Xiao G., Blanchi B., Sun Y.E. et al. (2010) $A$ selective trkb agonist with potent neurotrophic activities by 7,8-dihydroxyflavone. Proc. Nat. Acad. Sci. 107(6): 2687.

Jin Z., Chi M., He Q., Pan Y., Sun C. (2019) Perfluoroalkane sulfonyl fluorides non-covalently bind to human serum albumin at sudlow's sites. Toxicol. Lett. 301: 17-23.

Karimi M., Bahrami S., Ravari S.B., Zangabad P.S., Mirshekari H., Bozorgomid M., Shahreza S., Sori M., Hamblin M.R. (2016) Albumin nanostructures as advanced drug delivery systems. Expert Opin. Drug Deliv. 13(11): 1609-1623.

Khalili S., Zakeri A., Hashemi Z.S., Masoumikarimi M., Manesh M.R.R., Shariatifar N., Sani M.J. (2017) Structural analyses of the interactions between the thyme active ingredients and human serum albumin. Turkish J. Biochem. 42(4): 459-467.

Kim S., Thiessen P.A., Bolton E.E., Chen J., Fu G., Gindulyte A., Han L., He J., He S., Shoemaker B.A., et al. (2016) Pubchem substance and compound databases. Nucl. Acids Res. 44(D1): D1202-D1213.

Lee P., Wu X. (2015) Review: modifications of human serum albumin and their binding effect. Curr. Pharm. Des. 21(14): 1862-1865.

Liechti M.E. (2017) Modern clinical research on LSD. Neuropsychopharmacology 42(11): 2114-2127 (official publication of the American College of Neuropsychopharmaco$\log )$.

Ly C., Greb A.C., Cameron L.P., Wong J.M., Barragan E.V., Wilson P.C., Burbach K.F., Soltanzadeh Zarandi S., Sood A., Paddy M.R., et al. (2018) Psychedelics promote structural and functional neural plasticity. Cell Rep. 23(11): 3170-3182.

Matsuda R., Bi C., Anguizola J., Sobansky M., Rodriguez E., Vargas Badilla J., Zheng X., Hage B., Hage D.S. (2014) Studies of metabolite-protein interactions: a review. J. Chromatogr. B: Analyt. Technol. Biomed. Life Sci. 966: 48-58.

McConkey B.J., Sobolev V., Edelman M. (2002) The performance of current methods in ligand-protein docking. Curr. Sci. 83(7): 845-856.

Meloun B., Morávek L., Kostka V. (1975) Complete amino acid sequence of human serum albumin. FEBS Lett. 58(1): 134-137.

Meng X.Y., Zhang H.X., Mezei M., Cui M. (2011) Molecular docking: a powerful approach for structure-based drug discovery. Curr. Comp. Aided Drug Design 7(2): 146-157.

Nichols D.E. (2016) Psychedelics. Pharmacol. Rev. 68(2): 264-355.

Phillips A., Shaper G.A., Whincup P. (1989) Association between serum albumin and mortality from cardiovascular disease, cancer, and other causes. Lancet 334(8677): 1434-1436.

Purcell M., Neault J.F., Malonga H., Arakawa H., Carpentier R., Tajmir-Riahi H.A. (2001) Interactions of atrazine and 2,4-d 
with human serum albumin studied by gel and capillary electrophoresis, and ftir spectroscopy. Biochim. Biophys. Acta: Protein Struct. Mol. Enzymol. 1548(1): 129-138.

Ran D., Wu X., Zheng J., Yang J., Zhou H., Zhang M., Tang Y. (2007) Study on the interaction between florasulam and bovine serum albumin. J. Fluoresc. 17(6): 721-726.

Shalbafan M., Rezaei Behbehani G. (2018) Docking studies on the binding properties of methotrexate to human serum albumin. Biomacromol. J. 4(2): 114-117.

Sherwood A.M., Meisenheimer P., Tarpley G., Kargbo R.B. (2020) An improved, practical, and scalable five-step synthesis of psilocybin. Synthesis 52(05): 688-694.

Shooshtary S., Behtash S., Nafisi S. (2015) Arsenic trioxide binding to serum proteins. J.Photochem. Photobiol. B: Biol. 148: 31-36.

Silva D.l., Cortez C.M., Cunha-Bastos J., Louro S.R.W. (2004) Methyl parathion interaction with human and bovine serum albumin. Toxicol. Lett. 147(1): 53-61.

Sudlow G., Birkett D.J., Wade D.N. (1975) The characterization of two specific drug binding sites on human serum albumin. Mol. Pharmacol. 11(6): 824.

Swanson L.R. (2018) Unifying theories of psychedelic drug effects. Front. Pharmacol. 9: 172.
Tayyab S., Sam S.E., Kabir M.Z., Ridzwan N.F.W., Mohamad S.B. (2019) Molecular interaction study of an anticancer drug, ponatinib with human serum albumin using spectroscopic and molecular docking methods. Spectrochim. Acta A: Mol. Biomol. Spectrosc. 214: 199-206.

Trott O., Olson A.J. (2010) Autodock vina: improving the speed and accuracy of docking with a new scoring function, efficient optimization, and multithreading. J. Comput. Chem. 31(2): 455-461.

Uciechowska-Kaczmarzyk U., de Beauchene I.C., Samsonov S.A. (2019) Docking software performance in protein-glycosaminoglycan systems. J. Mol. Graph. Model. 90: 42-50.

Zielinski K., Sekula B., Bujacz A., Szymczak I. (2020) Structural investigations of stereoselective profen binding by equine and leporine serum albumins. Chirality 32(3): 334-344.

Zsila F. (2013) Subdomain ib is the third major drug binding region of human serum albumin: Toward the three-sites model. Mol. Pharma. 10(5): 1668-1682. 